\title{
Polydatin down-regulates the phosphorylation level of STAT3 and induces pyroptosis in triple-negative breast cancer mice with a high-fat diet
}

\author{
Min $\mathrm{Liu}^{1} \wedge$, Yinan $\mathrm{Li}^{2}$, Bingtan Kong ${ }^{2}$, Ganlin Zhang ${ }^{1}$, Qing Zhang ${ }^{1 \wedge}$ \\ ${ }^{1}$ Department of Oncology, Beijing Hospital of Traditional Chinese Medicine, Capital Medical University, Beijing, China; ${ }^{2}$ Beijing University of \\ Chinese Medicine, Beijing, China \\ Contributions: (I) Conception and design: M Liu, G Zhang, Q Zhang; (II) Administrative support: None; (III) Provision of study materials or patients: \\ None; (IV) Collection and assembly of data: None; (V) Data analysis and interpretation: M Liu, Y Li, B Kong; (VI) Manuscript writing: All authors; \\ (VII) Final approval of manuscript: All authors. \\ Correspondence to: Qing Zhang. Department of Oncology, Beijing Hospital of Traditional Chinese Medicine, Capital Medical University, 23 \\ Meishuguanhou Street, Dongcheng District, Beijing 100010, China. Email: zhangqing@ccmu.edu.cn; Min Liu. Department of Oncology, Beijing \\ Hospital of Traditional Chinese Medicine, Capital Medical University, 23 Meishuguanhou Street, Dongcheng District, Beijing 100010, China. \\ Email: liumin624@ccmu.edu.cn.
}

Background: To explore the impact of polydatin on mice with triple-negative breast cancer (TNBC) receiving a high-fat diet, as well as the underlying processes.

Methods: A total of 40 female Balb/c mice were randomly separated into 4 groups $(4 \mathrm{~T} 1+$ polydatin + fat diet group, 4T1 + high-fat diet group, 4T1 + polydatin group, and 4T1 group). To establish the obese TNBC mouse model, TNBC was xenografted $1 \times 10^{5} 4 \mathrm{~T} 1$ cells $/ 50 \mu \mathrm{L}$ per mouse at the right fourth mammary fat pad under anesthesia and the mice were fed a high fat diet. When the experiment was completed, total plasma cholesterol (TC) and cancer antigen (CA)15-3 were measured. The enzyme-linked immunosorbent assay (ELISA) method was used detect CA15-3. Oil red O staining was used to observe the morphological changes. Western blot analysis and reverse transcription polymerase chain reaction (RT-PCR) were used to detect the corresponding protein expression and the messenger RNA (mRNA) level.

Results: Polydatin decreased the degree of fatty liver, as determined by oil red $\mathrm{O}$ staining. The TC level in the 4T1 + fat diet group was significantly higher, and it was decreased in the 4T1 + polydatin group. The results of ELISA showed that compared with the 4T1 group, CA15-3 was significantly increased in the $4 \mathrm{~T} 1$ + fat diet group, and polydatin was shown to significantly reduce the expression of CA15-3. Polydatin inhibited p-JAK2 and p-STAT3 mRNA and protein levels. Polydatin increased pyroptosis-related gene mRNA and protein level.

Conclusions: We believe that polydatin can effectively reduce blood lipid levels in TNBC mice with a high-fat diet, and play an anticancer role in TNBC. The underlying mechanism may be related to the JAK2/ STAT3 signaling pathway and pyroptosis in TNBC. Our results contribute to validating the traditional use of polydatin in the treatment of TNBC with hyperlipidemia.

Keywords: Polydatin; triple-negative breast cancer (TNBC); JAK2/STAT3 signaling pathway; pyroptosis; highfat diet

Submitted Dec 17, 2021. Accepted for publication Feb 11, 2022.

doi: $10.21037 / \mathrm{atm}-22-73$

View this article at: https://dx.doi.org/10.21037/atm-22-73

^ ORCID: Min Liu, 0000-0002-2622-4091; Qing Zhang, 0000-0003-3713-4785. 


\section{Introduction}

Breast cancer (BC) is the most frequent kind of cancer in women and the most common contributor to cancer mortality. Among females, BC accounts for $11.7 \%$ of the total cancer incidences and $6.9 \%$ of the total cancer mortality (1). Treatment outcomes of BC have improved as a result of advances in surgery, chemotherapy, and hormone therapy. However, there is currently no effective treatment for triple-negative breast cancer (TNBC), which has been shown not to express genes for the progesterone receptor (PR), the epidermal growth factor receptor 2 (Her2), or estrogen receptor (ER).

Mammary adenocarcinoma develops in a fat-rich milieu, which has been demonstrated to be a key player in the progression of the tumor (2). Obesity is a recognized risk factor for mammary adenocarcinoma (3). Cancerassociated adipocytes (CAA) are formed as a result of interactions between cancer cells and adipocytes in the tumor microenvironment. It has been shown that CAA undergo morphological alterations and develop new abilities that may influence the evolution of BC. Polydatin is a naturally occurring active substance derived from the root of Polygonum cuspidatum. It has pharmacological effects in a variety of medical domains, for example, it suppresses inflammation in hepatocytes (4). In recent years, polydatin has been shown to have anti-tumor effects in many cancers. It can cause cell death by weakening the phosphorylation of Creb (5). In colon cancer, polydatin could increase cell apoptosis by calcium influx to fight 5 -fluorouracil resistance (6). Previous research has shown that polydatin has antiinflammatory properties in mature adipocyte cells. In mice given a high-fat diet, polydatin suppresses adipose tissue inflammation and improves lipid metabolism (7).

According to current research, BC is primarily associated with the following pathways: immunizing inflammatory response, oxidative stress, and the treatment for BC is focused on immunity and inducing cell pyroptosis and apoptosis. One of the most important signaling pathways in cell metabolism and apoptosis is the Janus kinase/ signal transduction and transcription activator (JAK/ STAT) signaling pathway. Previous studies have shown that STAT3 could proliferate BC cells by promoting glycolysis (8). Activation of STAT3 in mammary cancer cells modulates mitochondrial electron transport chains and promotes mammary tumor growth in vivo (9). Recent studies have shown that obese women are more likely to develop malignant TNBC without treatment (10).
However, few reports have shown how polydatin impacts on mice TNBC with a high-fat diet. In this study, we used Balb/c mice to investigate the mechanisms by which a high-fat diet stimulates the formation of xenografts from TNBC. We hypothesized the phosphorylation level of STAT3 inhibition and pyroptosis activation would be a new anticancer mechanism of polydatin in mammary cancer cells and investigated the JAK2/STAT3 signaling pathway in TNBC mice fed with a high-fat diet to trigger pyroptosis. We present the following article in accordance with the ARRIVE reporting checklist (available at https://atm. amegroups.com/article/view/10.21037/atm-22-73/rc).

\section{Methods}

\section{Animals used in experiments}

The current investigation involved female Balb/c mice that were 8 weeks old (Beijing HFK Bioscience Co., Ltd., Beijing, China). In accordance with standard laboratory procedures, the animal models were established in Beijing Viewsolid Biotechnology Co., Ltd., and all animals were housed at the Beijing Hospital of Traditional Chinese Medicine $\left(22-24{ }^{\circ} \mathrm{C}, 40-60 \%\right.$ relative humidity) food and water were freely available, and the light/dark-cycle was maintained for 12/12 hours, with the light turned on at 6:00 am. All experimental processes were carried out in compliance with the Guidelines for the Care and Use of Laboratory Animals, which were developed by the Chinese Ministry of Science and Technology (Beijing, China). Experiments were performed under a project license granted by ethics board of Beijing Viewsolid Biotechnology Co., Ltd. (No. 202000038).

\section{Polydatin therapy in an animal model}

Female Balb/c mice were divided into 4 groups $(n=10$ in each group) according to their body weight, as shown below: (I) a standard chow meal was provided to the 4T1 group (10\% kcal fat, $70 \%$ kcal carbohydrates, and $20 \%$ kcal protein); (II) a high-fat diet (45\% kcal fat, 35\% kcal carbohydrates, and $20 \% \mathrm{kcal}$ proteins) was provided to the $4 \mathrm{~T} 1$ + high-fat diet group; (III) $4 \mathrm{~T} 1$ + polydatin treatment group was fed a regular chow diet and polydatin $100 \mathrm{mg} / \mathrm{kg}$ by intraperitoneal injection daily (11); (IV) 4T1 + polydatin + fat diet group was fed with a high-fat diet and polydatin $100 \mathrm{mg} / \mathrm{kg}$ intraperitoneal injection daily. All mice were subcutaneously xenografted with $1 \times 10^{5} 4 \mathrm{~T} 1$ cells/50 $\mu \mathrm{L}$ 
each in the right fourth mammary fat pad under anesthesia. The animal procedures of observed indicators followed the methods of Abdelwahed et al., 2020 (12).

Subsequently, we dissolved polydatin in dimethylsulfoxide (DMSO) in order to yield an end product with a $1 \mathrm{mM}$ stock solution that could be used to prepare treatment media at various concentrations. All treatment groups had constant end DMSO concentrations in the process of the experiment, with no group exceeding $0.1 \%$ of their initial concentrations.

\section{Examination of the effects of polydatin on the total cholesterol level in the blood}

Total plasma cholesterol (TC) was determined in polydatintreated and control mice using a total plasma cholesterol test kit (Nanjing Jiancheng Bioengineering Institute, Nanjing, China) in accordance with the guidelines provided by the manufacturer.

\section{Impact of polydatin therapy on plasma levels of the $B C$ metastasis marker cancer antigen 15-3}

The impact of polydatin on the recurrence and metastasis marker of BC was determined using the mouse carbohydrate antigen 15-3 enzyme-linked immunosorbent assay (ELISA) kit (Krishgen BioSystems, Mumbai, India) according to the guidelines provided by the manufacturer.

\section{Staining using Oil Red O}

Fixation of the livers was performed for $24 \mathrm{~h}$, followed by dehydration in $15 \%$ and $30 \%$ sucrose solutions at $-4{ }^{\circ} \mathrm{C}$ in succession. Then, the livers were optimal cupping temperature (OCT) embedded, cold-sliced $(8 \mu \mathrm{m})$, refixed at ambient temperature for an additional $20 \mathrm{~min}$, and stained with Oil Red O for $20 \mathrm{~min}$. Then, 75\% alcohol was used to differentiate the livers and hematoxylin was used to counterstain them for 2 min. Finally, the livers were covered using neutral balsam.

\section{Western blot analysis}

The routine processes for western blotting were carried out in the same manner as previously described (13). The following primary antibodies were used: Rabbit anti-STAT3 antibody (1:1,000, Abcam, Cambridge, USA), Rabbit antiJAK2 antibody [1:1,000, Cell Signaling Technology (CST),
Danvers, MA, USA], rabbit anti-Phospho-STAT3 antibody (1:500, CST, USA), rabbit anti-IL1 $\beta$ antibody (1:500, Abcam, USA), rabbit anti-Phospho-JAK2 antibody (1:500, CST, USA), rabbit anti-pro Caspase- $1+$ p10 + p12 antibody (1:500, Abcam, USA), rabbit anti-Cleaved Caspase-3 antibody (1:1,000, CST, USA), rabbit anti-NLRP3 antibody (1:800, Abcam, USA), rabbit anti-IL18 antibody (1:500, Abcam, USA), rabbit anti-Caspase- 3 antibody (1:1,000, CST, USA), rabbit anti-Caspase-1 antibody (1:500, Abcam, USA), rabbit anti-GSDMD antibody (1:500, Abcam, USA), rabbit anti-GAPDH antibody $(1: 3,000$, Abcam, USA), and mouse $\beta$-actin monoclonal antibody $(1: 3,000$, Abcam, USA). Subsequently, blots were rinsed thrice in Tris-buffered saline containing 0.1 percent Tween-20 (TBST). Following rinsing with TBST, DyLight ${ }^{\mathrm{TM}} 680$ or 800 -labeled secondary antibody $(1: 10,000)$ was used to incubate the blots for $1 \mathrm{~h}$, followed by a rinsing in TBST 3 times. Odyssey version 3.0 (LI-COR Biosciences, Lincoln, NE, USA) was used to determine the relative density of the protein band samples. Image-Pro Plus Version 6.0 software (Media Cybernetics, Rockville, MD, USA) was used to determine the signal strength.

\section{Quantitative real-time polymerase chain reaction}

The total messenger RNA (mRNA) from the tissues was obtained. A total of 2,000 ng mRNA was converted into complementary DNA (cDNA). The quantitative analysis of target genes and the reference gene (GAPDH) was carried out in triplicate on the CFX96 Touch (Bio-Rad Inc., Hercules, CA, USA). The primers used were as follows: STAT3 forward 5'-TATGGTCCTTATTCTATGCG-3' and reverse 5'-CAGACAGTTGCCAGTCTCA-3'; 7AK2 forward 5'-CTTCCACATAGACGAGTCAACCA-3' and reverse 5'-GTTCTGCTGCTGCCACTACA-3'; NLRP3 forward 5'-ATTACCCGCCCGAGAAAGG-3' and reverse 5'-TCGCAGCAAAGATCCACACAG-3'; Caspase-1 forward 5'-ACAAGGCACGGGACCTATG-3' and reverse 5'-TCCCAGTCAGTCCTGGAAATG-3'; $I L-1 \beta$ forward 5'-CGCAGCAGCACATCAACAAGAGC-3' and reverse 5'-TGTCCTCATCCTGGAAGGTCCACG-3'; IL 18 forward 5'-GACCAAGTTCTCTTCGTTGACAA-3' and reverse 5'-ACAGCCAGTCCTCTTACTTCAC-3'; GSDMD forward 5'-CCATCGGCCTTTGAGAAAGTG-3' and reverse 5'-ACACATGAATAACGGGGTTTCC-3'; Caspase-3 forward 5'-AGCACCTGGTTACTATTCCTG-3' and reverse 5'-ATTCCGTTGCCACCTTCC-3'; GAPDH forward 5'-AGGTCGGTGTGAACGGATTTG-3' and 
A

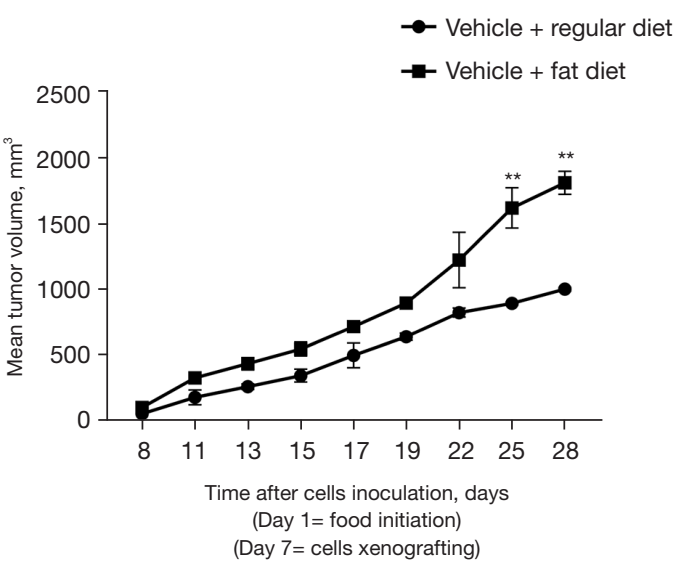

B

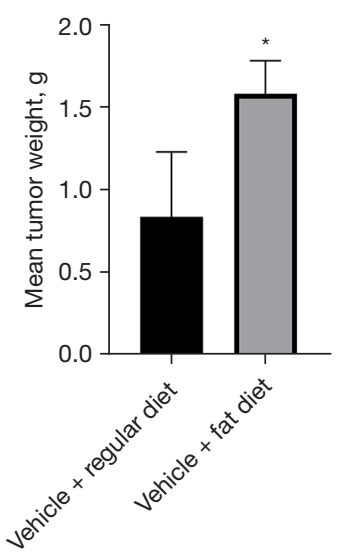

Figure 1 Using an orthotopic Balb/c mouse xenograft model, we investigated whether receiving a high-fat diet or eating regularly would have any influence on the occurrence and progression of TNBC. (A) Comparison of the average tumor volume of 4T1 cells. The mean tumor volumes for each experimental group are shown by the points on the graph. (B) Weight comparisons of 4T1 cell tumors on an average basis. The SEM of each experimental group is shown by error bars. ${ }^{*} \mathrm{P}<0.05$ and ${ }^{* *} \mathrm{P}<0.01$ were used to indicate statistical significance when compared to the regular chow diet-fed control group. TNBC, triple-negative breast cancer; SEM, standard error of the mean.

reverse 5'-TGTAGACCATGTAGTTGAGGTCA-3'. The target gene expression levels were normalized to GAPDH and determined using the comparative threshold cycle technique.

\section{Statistical analysis}

The mean \pm standard error of the mean $($ SEM) of at least 2 separate experiments was used to express the results. Student's 2 -tailed $t$-test was used to determine the differences between the 2 groups. We used one-way analysis of variance (ANOVA) to identify the differences among 3 or more groups, followed by Dunnett's post hoc analysis. GraphPad Prism software (version: 8.0; GraphPad Inc., La Jolla, CA, USA) was used to complete all statistical analyses. Significant differences were considered when ${ }^{*} \mathrm{P}<0.05$ and ${ }^{* *} \mathrm{P}<0.01$.

\section{Results}

\section{A bigh-fat diet promoted the progression of 4T1 TNBC in a breast tumor-bearing mice model}

The purpose of this study was to assess the impact of a highfat diet against a standard chow diet on the progression of TNBC in a Balb/c mouse model of breast tumors. For the mice given a standard chow diet, the mean tumor volume was $1,014 \pm 19.36 \mathrm{~mm}^{3}$. For mice given a high-fat diet, the mean tumor volume was $1,823.33 \pm 19.85 \mathrm{~mm}^{3}$, about 1.8 times that of animals on a standard diet (Figure 1A). In contrast with the normal diet group, the high-fat diet group had a statistically significant elevation in the mean tumor weight of $1.6 \pm 0.32 \mathrm{~g}$, while the mice in the normal diet group had a mean tumor weight of $0.85 \pm 0.22 \mathrm{~g}$ (Figure $1 B$ ). When compared to mice fed a normal chow diet, those given a high-fat diet had thrice the weight of tumors.

\section{Polydatin inbibited the progression of TNBC in breast tumor-bearing mice model}

A $100 \mathrm{mg} / \mathrm{kg}$ daily intraperitoneal injection polydatin regimen administered to Balb/c mice fed a high-fat diet and harboring 4T1 tumor cells model of xenograft was tested for anti-tumor efficacy. The 4T1 + high-fat diet group was utilized as a substitute for the $4 \mathrm{~T} 1$ group. Polydatin treated mice had a mean tumor volume of $336.33 \pm 16.21 \mathrm{~mm},{ }^{3}$ which was significantly higher compared to $1,014 \pm 19.36 \mathrm{~mm}^{3}$ previously reported in the $4 \mathrm{~T} 1$ group (Figure $2 A$ ). In comparison with the 4T1 group, polydatin therapy dramatically inhibited the progression of $4 \mathrm{~T} 1$ tumors by $66.9 \%$. The average weight of the tumors for $4 \mathrm{TI}$ was $0.85 \pm 0.22 \mathrm{~g}$, and $0.31 \pm 0.18 \mathrm{~g}$ for the $4 \mathrm{~T} 1+$ polydatin group animals (Figure $2 B$ ). 
A

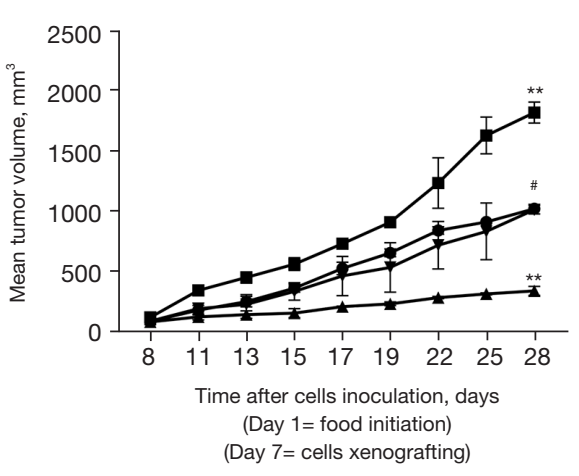

B

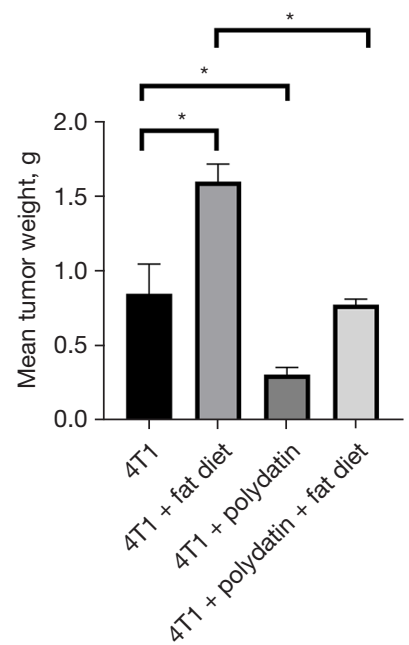

Figure 2 Polydatin therapy has an effect on the development of orthotopically xenografted TNBC cells 4T1 in high-fat Balb/c mice. (A) Comparative monitoring of the mean $4 \mathrm{~T} 1$ cells tumor volume for the $4 \mathrm{~T} 1$ + polydatin group $v s$. the $4 \mathrm{~T} 1$ group during the course of the trial. The points denote the mean tumor volumes, while the error bars denote the standard error of the mean for each of the experimental groups. ${ }^{*} \mathrm{P}<0.05$ indicates statistical significance in comparison with the $4 \mathrm{~T} 1+$ polydatin control group. ${ }^{* *} \mathrm{P}<0.01$ indicates statistical significance in comparison with the 4T1 group. (B) At the later stage of the trial, the mean value of tumor weights was compared between the $4 \mathrm{~T} 1+$ polydatin and $4 \mathrm{~T} 1$ groups. Error bars represent the SEM. ${ }^{*} \mathrm{P}<0.05$ is considered statistically significant. TNBC, triple-negative breast cancer; SEM, standard error of the mean.

\section{Polydatin inbibited lung metastasis and decreased plasma TC level}

To determine the effect of a high-fat diet on tumor development, we constructed orthotopic breast cancer models. In comparison with the 4T1 group, the 4T1 + high-fat diet group had a greater tumor burden (Figure 2A) and more significant lung metastasis (200.33 vs. $147.67 \mathrm{~g}$ ) (Figure 3A). Cancer antigen 15-3 (CA15-3) is a marker for human breast cancer recurrence and metastasis that was detected in the plasma of Balb/c mice at the later stage of the experiment. The CA15-3 plasma levels were considerably greater in the 4T1 mice group than in the $4 \mathrm{~T} 1$ + polydatin group mice, representing an $81.8 \%$ decrease in the metastatic indicator CA15-3 in the therapy group (Figure 3B).

We assessed mice that had been given a high-fat diet. As indicated by histology, a high-fat diet eventually led to liver damage and steatosis (Figure 3C). The TC levels were decreased in polydatin treated mice $(83.67 \pm 8.45 \mathrm{mg} / \mathrm{dL})$ compared to the $4 \mathrm{~T} 1$ group $(135.033 \pm 10.56 \mathrm{mg} / \mathrm{dL})$, which resulted in a $38.04 \%$ drop in TC level for the $4 \mathrm{~T} 1+$ polydatin group (Figure 3D).

\section{In BC tissues, polydatin suppressed the $\mathcal{F A K 2}$-STAT3 signaling pathway}

Using TNBC mice fed a high-fat diet, we further studied the mechanism underlying polydatin anti-BC. We detected the JAK2 and STAT3 mRNA expressions in BC tissues by reverse transcription polymerase chain reaction (RTPCR) and studied the JAK2 and STAT3 protein expressions using western blot. Polydatin significantly inhibited JAK2 and STAT3 mRNA expression (Figure 4A), respectively, and significantly inhibited protein expressions of p-STAT3 and p-JAK2 (Figure 4B). The results showed that polydatin inhibits the JAK2-STAT3 signaling pathway in mice TNBC with a high-fat diet.

\section{Polydatin promoted pyroptosis of BC cells in tissues}

Pyroptosis requires caspase- 1 activation which converts the cytokines interleukin 18 (IL-18) and IL- $1 \beta$ into their active forms and subsequently induces pyroptotic cell apoptosis. Activation of Caspase-1 needs a protein platform called inflammasome and the NLRP3 inflammasome has been widely investigated. In the present study, we obtained BC 
A

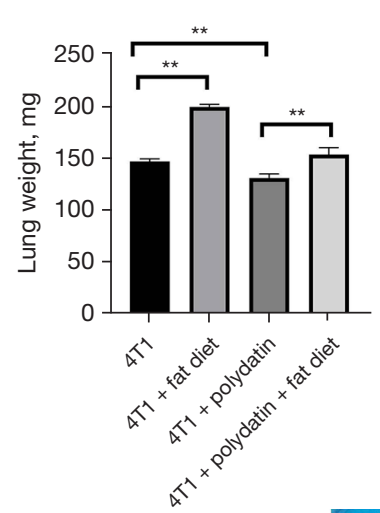

B

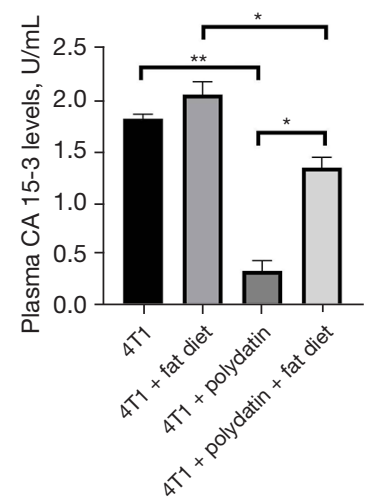

$\mathrm{D}$

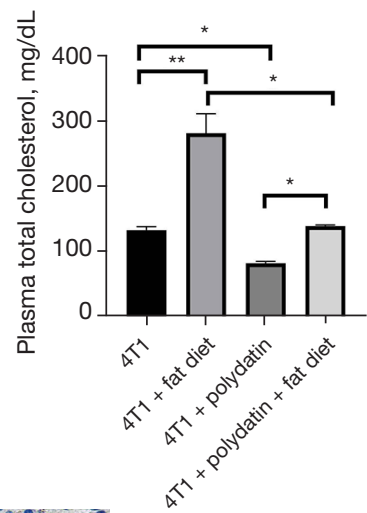

C
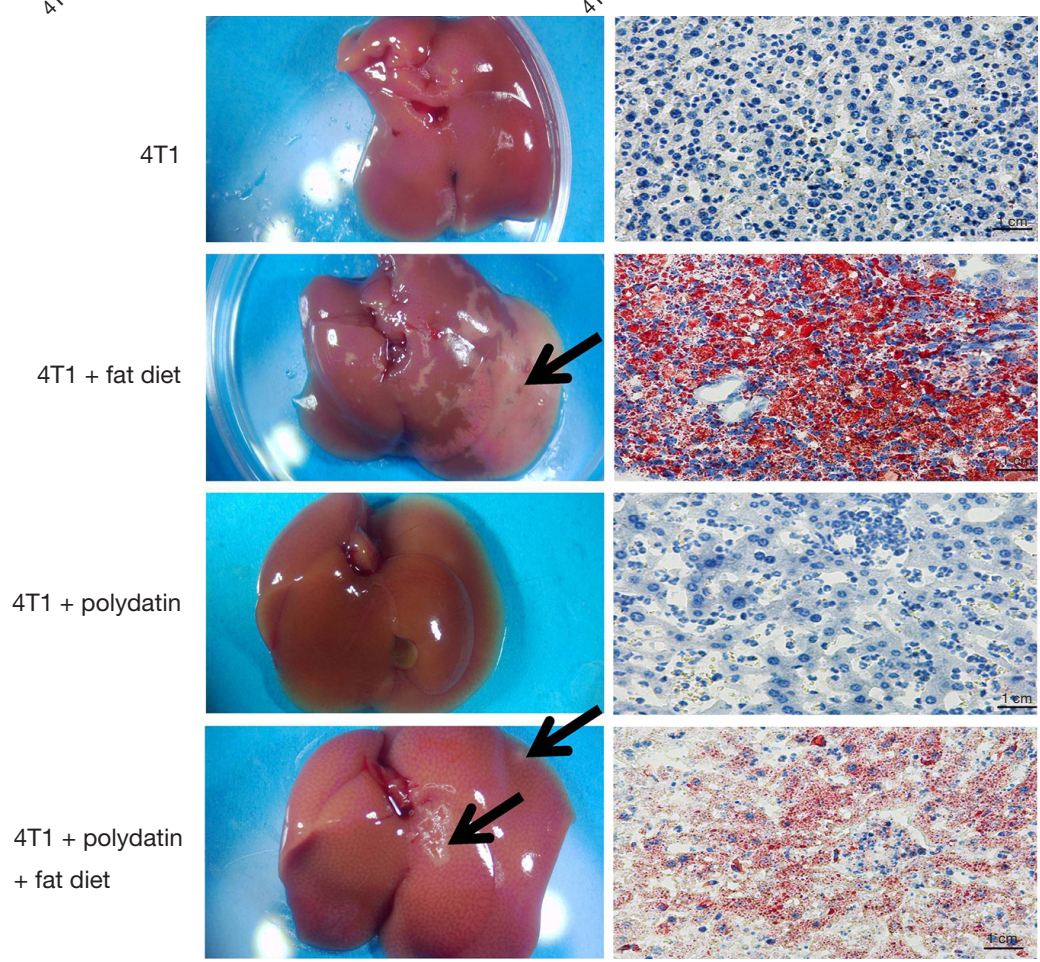

Figure 3 Polydatin therapy effects on lung weight, CA15-3 level, fatty liver, and plasma TC in 4T1 tumors in Balb/c mice receiving a high-fat diet. (A) At the later stage of the experiment, we compared the mean lung weight of the 4T1 + polydatin group with that of the 4T1 group in an orthotopic Balb/c mice xenograft model. (B) The plasma levels of the breast cancer recurrence marker CA15-3 in the 4T1 + polydatin group were compared to those in the $4 \mathrm{~T} 1$ group in an orthotopic Balb/c mice xenograft model. (C) The degree of fatty liver (arrow) was compared between the 4T1 + polydatin and 4T1 groups in an orthotopic Balb/c mouse xenograft model. The liver tissues were stained with Oil Red O. Magnification in its original state is $\times 400$. (D) The mean plasma TC levels in the T1 + polydatin group were compared to those in the 4T1 group in $4 \mathrm{~T} 1$ tumors. ${ }^{*} \mathrm{P}<0.05$ and ${ }^{*} \mathrm{P}<0.01$ are considered statistically significant. CA15-3, cancer antigen $15-3$; $\mathrm{TC}$, total cholesterol.

RNA from breast tissue for RT-PCR analysis to explore if the expression of pyroptosis-related genes was changed in mice receiving a high-fat diet. Notably, as displayed in Figure 5A, IL-18, IL-1 $\beta$, GSDMD, Caspase-1, NLRP3, and Caspase-3 expressions were considerably reduced in the RNA samples of mice receiving a high-fat diet. The expression levels of these genes decreased in mice that were treated with polydatin. As illustrated in Figure 5B, proCaspase-1, IL-1 $\beta$, Caspase-3, NLRP3, IL-18, Cleaved Caspase- 3 , and Caspase- 1 in the protein samples were also significantly reduced in mice receiving a high-fat diet. In the $4 \mathrm{~T} 1+$ polydatin group, these protein expressions showed 
A

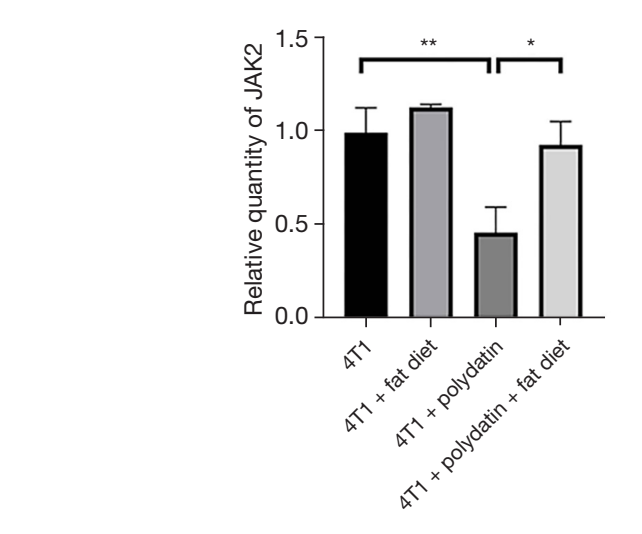

B
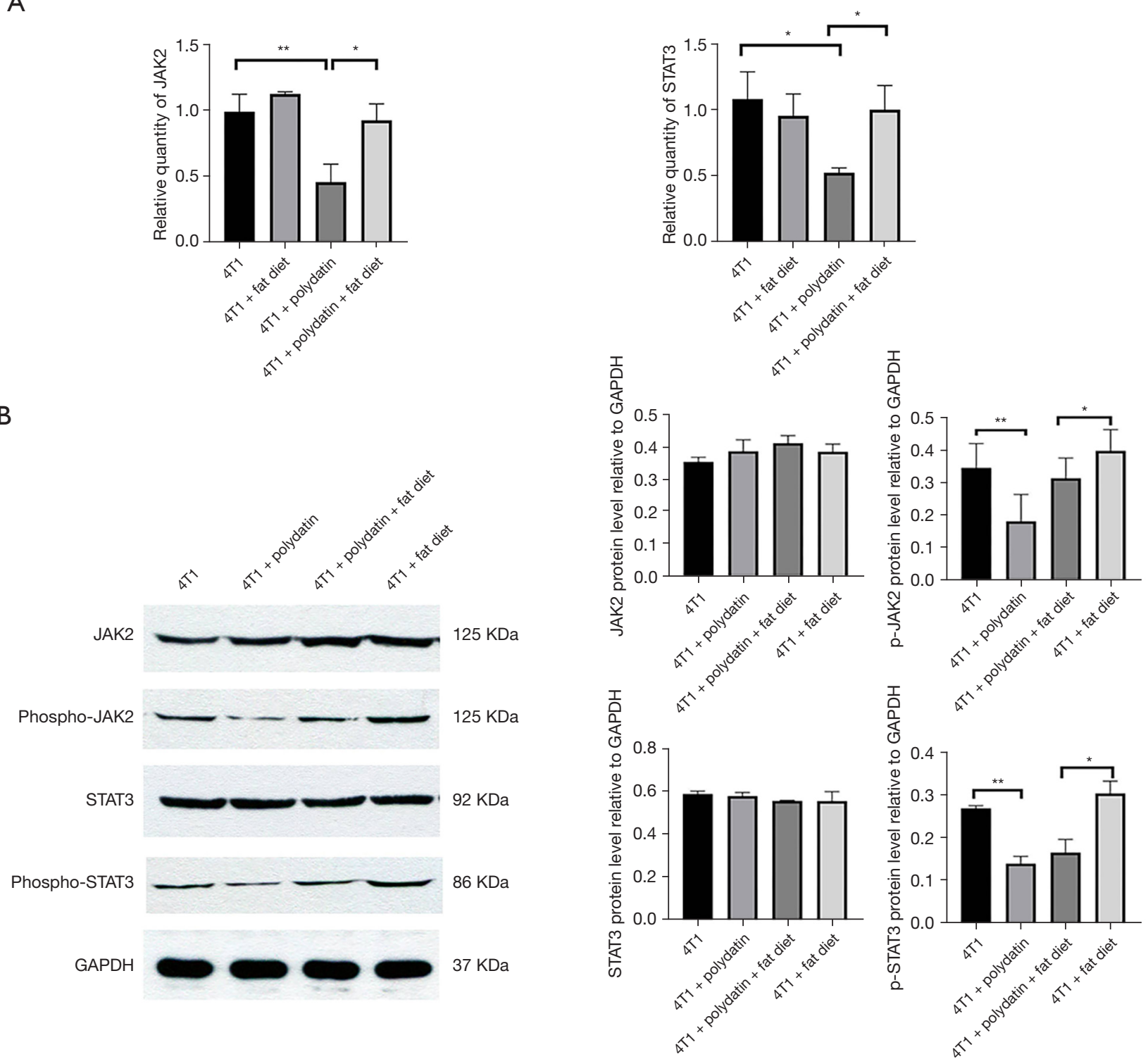

Figure 4 The impacts of polydatin treatments on JAK2 and STAT3 expression levels. (A) STAT3 and JAK2 mRNA expressions in breast cancer tissues treated with 4T1, 4T1 + polydatin, 4T1 + polydatin + fat diet, and 4T1 + fat diet. (B) STAT3, JAK2, phospho-STAT3, and phospho-JAK2 protein expressions in BC tissues treated with 4T1, 4T1 + polydatin, 4T1 + polydatin + fat diet, and 4T1 + fat diet. All blots were subjected to scanning densitometry in triplicate, and the integrated optical density of each band was standardized to that of GAPDH in the same blot. ${ }^{*} \mathrm{P}<0.05,{ }^{* *} \mathrm{P}<0.01$ was considered statistically significant. mRNA, messenger RNA; BC, breast cancer.

a downward trend. Notably, there were no statistically significant differences in the expression levels of GSDMD protein across the 4 groups,

\section{Discussion}

Female $\mathrm{BC}$ is the most frequent kind of malignancy.
Compared with other subtypes of BC, patients with metastatic TNBC have a median overall survival of roughly 18 months (14). In recent years, an important topic of investigation has been the relationship between the progression of mammary adenocarcinoma and the presence of breast adipose tissue or a tumor microenvironment (15). Adipose tissue is an endocrine organ that produces a 

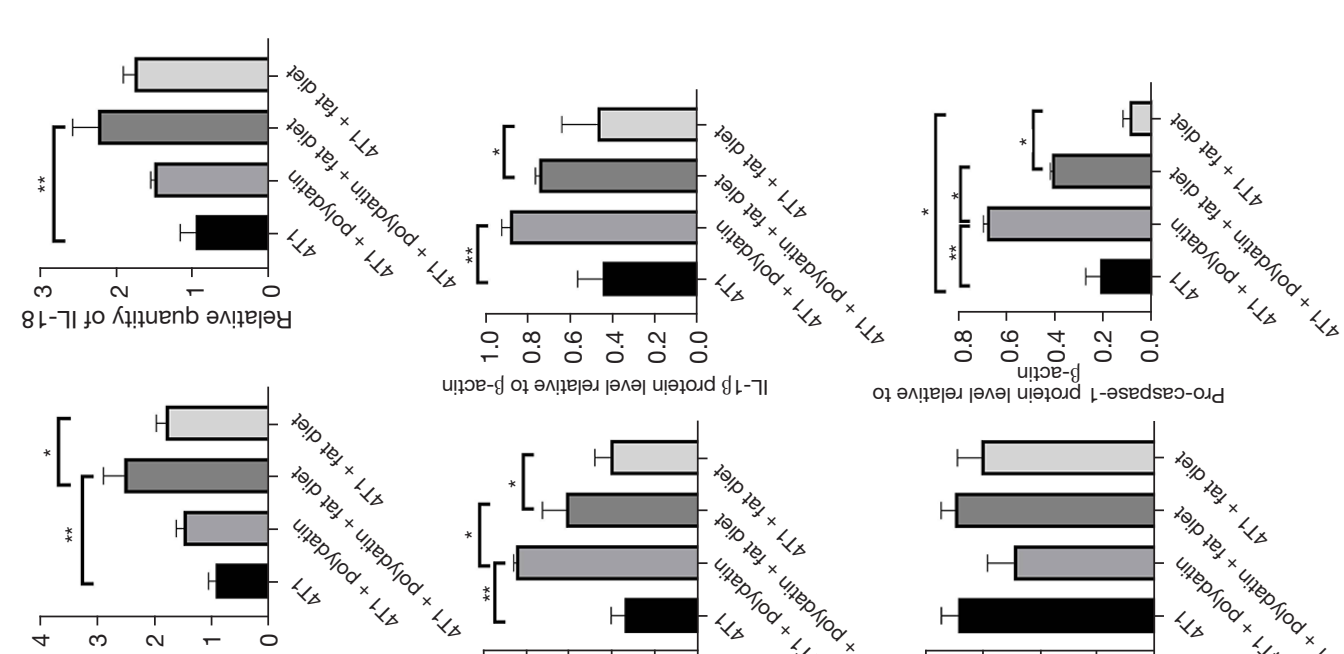

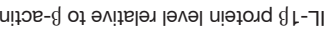

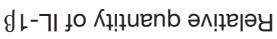

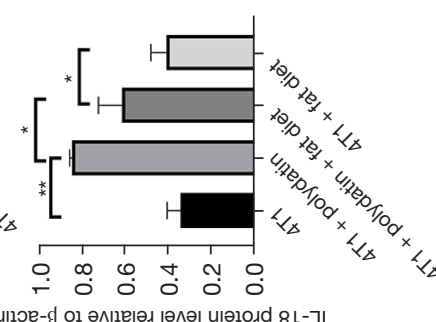

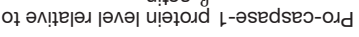

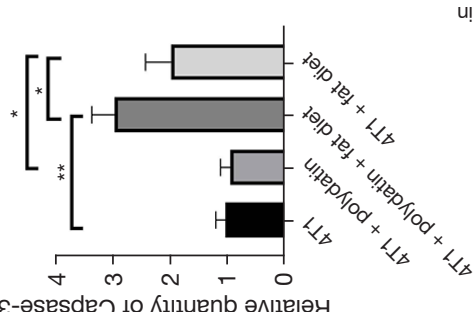

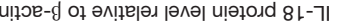
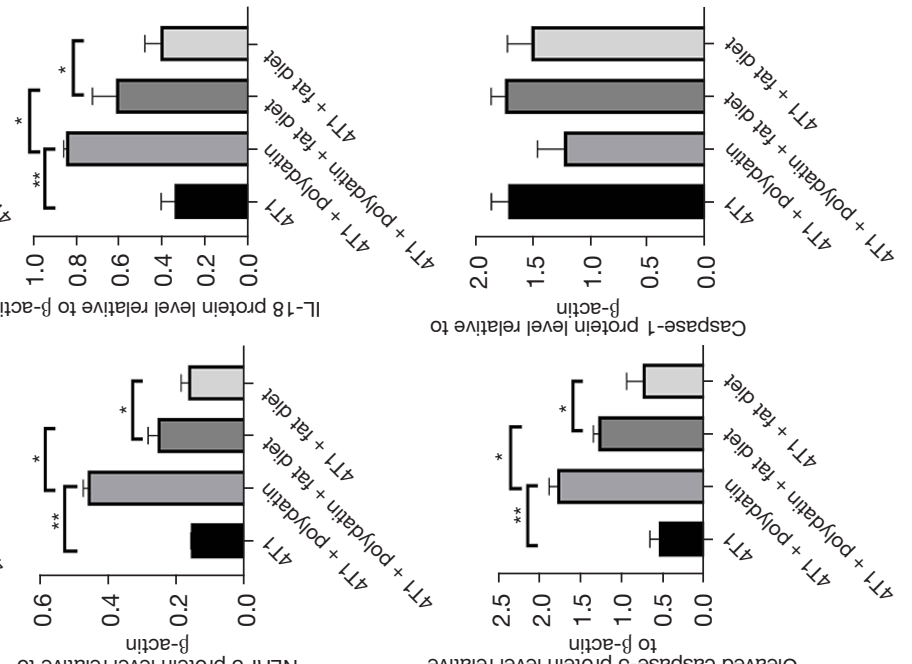

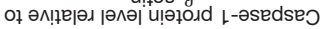
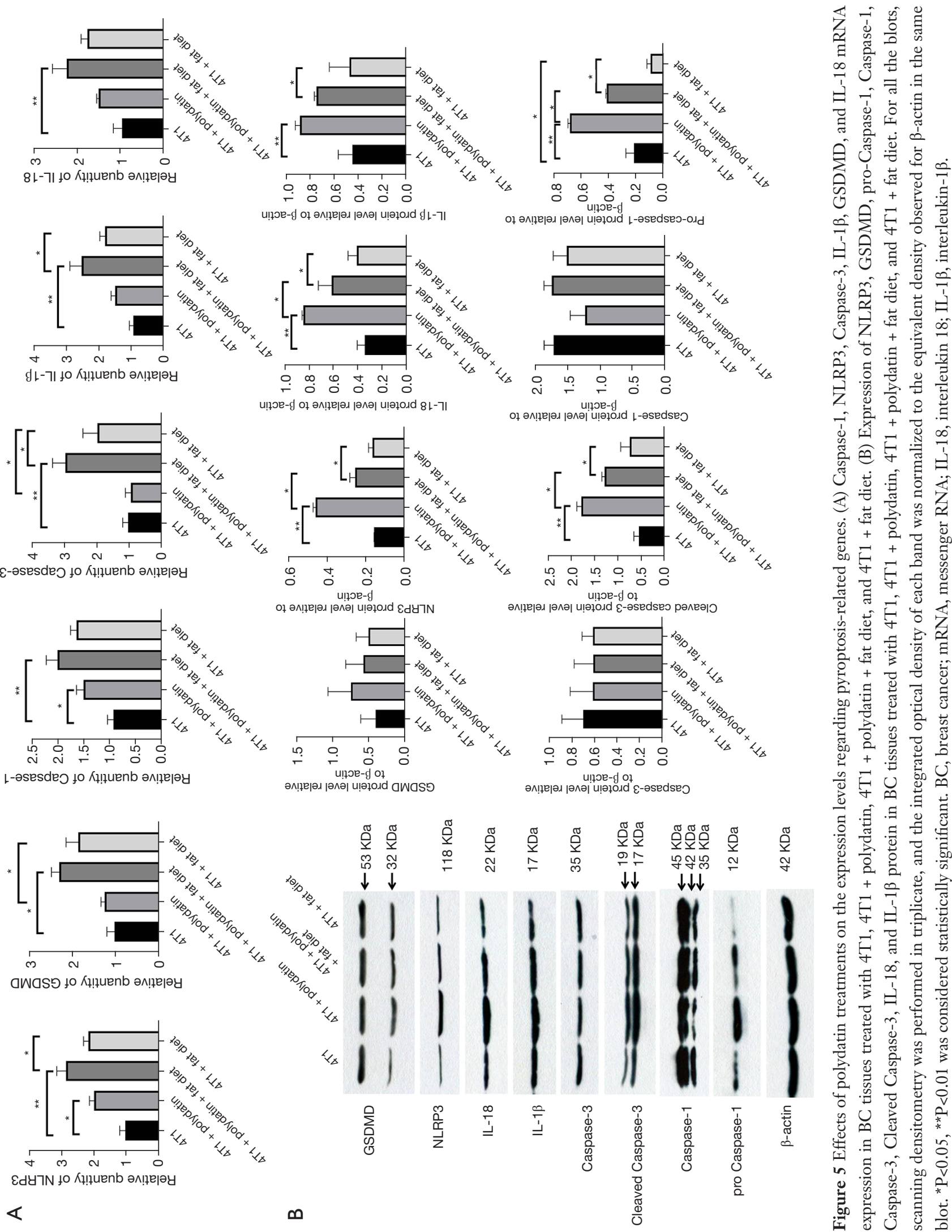

1. 
number of substances, such as bioactive lipids, that influence both systemic and local metabolic signals. Specific metabolic alterations in adipose tissue in the mammary glands are related to the progression of mammary adenocarcinoma via establishment of a tumor-causing microenvironment $(16,17)$. Furthermore, obesity itself has a significant impact on adipocyte metabolism and is an important risk factor for the occurrence and progression of TNBC (18). Polydatin is a new herbal anticancer medication that has been shown to be therapeutically efficacious in the treatment of several malignancies, particularly $\mathrm{BC}$ associated with hyperlipidemia. In terms of anticancer activity, polydatin has a wide range $(19,20)$ : it may be used in a variety of cytotoxic medicines, such as doxorubicin, to have a synergetic impact (21), and also enhance the chemosensitivity of cancer cells to paclitaxel (22).

Previous study has shown that emodin could inhibited tumor growth, and suppressed the lung and liver metastasis of TNBC cells by decreasing the secretion of CCL5 in mice fed a high fat diet (23). Another study showed that Moringa supplementation protected against high-fat diet, and Moringa supplementation may be an effective strategy to improve metabolic health in mice with obesity and TNBC (24). In our mouse model, polydatin potently decreased distant metastases and reduced fatty liver and plasma TC. Meanwhile, polydatin boosted Caspase-1, Caspase-3, IL-1 $\beta$, NLRP3, and IL-18 protein expression that participate in pyroptosis occurrence. Furthermore, downmodulation of STAT3 and JAK2 phosphorylation played a critical function in these processes. However, the research on polydatin's anti-breast cancer role is less, just one study found that 2-DG and polydatin both inhibited cell viability in 4T1 and MCF-7 and that PD combined with 2-DG obviously induced apoptosis and led to a reduction in cell proliferation and migration in 4T1 and MCF-7 compared to the individual treatment groups (11). While our findings showed that polydatin may have an anti-cancer effect by triggering pyroptosis, which is dependent on the JAK2/ STAT3 signaling pathway. The effects of polydatin on mice TNBC with a high-fat diet in experimental models were reported for the first time in the present study, according to our understanding, via the JAK2/STAT3 signaling pathway.

Pyroptosis is closely related to tumor progression, tumor prognosis, and immunotherapy response. Evaluating the Pyroptosis Score of a single tumor can assist in understanding the characteristics of TME infiltration and lead to the development of more effective immunotherapy strategies (25). Previous research shown that the RS was distinctly cancer immunity cycle, HLA immune cell infiltration and immune checkpoints in breast cancer. Pyroptosis combining with immunotherapies might be a potential therapeutic strategy (26).Currently, extensive study is being conducted into the pathophysiology of TNBC. Various investigations have shown that several herbal medications may block the STAT3 signaling pathway, as well as cell migration, invasion, and viability in TNBC $(27,28)$. Cisplatin can induce pyroptosis via the NLRP3/caspase-1/GSDMD pathway in TNBC (29). Polydatin, according to our findings, induces apoptosis in TNBC by increasing the activation of caspase- 3 and lowering the phosphorylation of JAK2 and STAT3 in the cell. Cell death and the release of inflammatory factors are caused by GSDMD formation of plasma membrane holes in TNBC. These findings of the present study are in line with those of previous research. Thus, we reached the conclusion that cell apoptosis and pyroptosis play essential roles in the progression of TNBC in the presence of a high-fat diet.

According to mounting information, the JAK/STAT signaling system participates in a growing number of disorders (30-32). To our knowledge, no prior research had been conducted to evaluate the role of JAK2/STAT3 signaling in TNBC associated with a high-fat diet. Phosphorylation activation of JAK2/STAT3 has been reported as a trigger for $\mathrm{BC}$ stem cells and a new prognostic indicator for BC metastasis to various organs (33). In the present investigation, we found that reduced phosphorylation of JAK2/STAT3 increased pyroptosis and apoptosis in TNBC cells in vivo. Further investigation into the effects of polydatin on pyroptosis and apoptosis is needed in the future to identify additional therapeutic targets and to enhance the practical application of polydatin for TNBC in patients receiving a high-fat diet.

\section{Conclusions}

The results of the present study indicate that polydatin decreases distant metastases and reduces fatty liver and plasma TC. Specifically, polydatin was shown to enhance apoptosis as well as pyroptosis by inhibiting the JAK2/STAT3 signaling pathway. The findings of this research are encouraging, and they must be validated in order to investigate the potential of employing polydatin as a treatment agent for TNBC associated with hyperlipidemia in the future.

\section{Acknowledgments}

Funding: This study was funded by Enshi Prefecture Science 
and Technology Program Research and Development Project (No. JCY2019000040). The funders had no stakes in the design, collection of data and analysis, decisionmaking of publishing, or the writing of the manuscript.

\section{Footnote}

Reporting Checklist: The authors have completed the ARRIVE reporting checklist. Available at https://atm. amegroups.com/article/view/10.21037/atm-22-73/rc

Data Sharing Statement: Available at https://atm.amegroups. com/article/view/10.21037/atm-22-73/dss

Conflicts of Interest: All authors have completed the ICMJE uniform disclosure form (available at https://atm. amegroups.com/article/view/10.21037/atm-22-73/coif). The authors have no conflicts of interest to declare.

Etbical Statement: The authors are accountable for all aspects of the work in ensuring that questions related to the accuracy or integrity of any part of the work are appropriately investigated and resolved. All experimental processes were carried out in compliance with the Guidelines for the Care and Use of Laboratory Animals, which were developed by the Chinese Ministry of Science and Technology (Beijing, China). Experiments were performed under a project license granted by ethics board of Beijing Viewsolid Biotechnology Co., Ltd. (No. 202000038).

Open Access Statement: This is an Open Access article distributed in accordance with the Creative Commons Attribution-NonCommercial-NoDerivs 4.0 International License (CC BY-NC-ND 4.0), which permits the noncommercial replication and distribution of the article with the strict proviso that no changes or edits are made and the original work is properly cited (including links to both the formal publication through the relevant DOI and the license). See: https://creativecommons.org/licenses/by-nc-nd/4.0/.

\section{References}

1. Sung H, Ferlay J, Siegel RL, et al. Global Cancer Statistics 2020: GLOBOCAN Estimates of Incidence and Mortality Worldwide for 36 Cancers in 185 Countries. CA Cancer J Clin 2021;71:209-49.

2. Lengyel E, Makowski L, DiGiovanni J, et al. Cancer as a Matter of Fat: The Crosstalk between Adipose Tissue and
Tumors. Trends Cancer 2018;4:374-84.

3. Picon-Ruiz M, Morata-Tarifa C, Valle-Goffin JJ, et al. Obesity and adverse breast cancer risk and outcome: Mechanistic insights and strategies for intervention. CA Cancer J Clin 2017;67:378-97.

4. Chen X, Chan H, Zhang L, et al. The phytochemical polydatin ameliorates non-alcoholic steatohepatitis by restoring lysosomal function and autophagic flux. J Cell Mol Med 2019;23:4290-300.

5. Chen S, Tao J, Zhong F, et al. Polydatin down-regulates the phosphorylation level of $\mathrm{Creb}$ and induces apoptosis in human breast cancer cell. PLoS One 2017;12:e0176501.

6. Bae H, Lee W, Song J, et al. Polydatin Counteracts 5-Fluorouracil Resistance by Enhancing Apoptosis via Calcium Influx in Colon Cancer. Antioxidants (Basel) 2021;10:1477.

7. Zheng L, Wu J, Mo J, et al. Polydatin Inhibits Adipose Tissue Inflammation and Ameliorates Lipid Metabolism in High-Fat-Fed Mice. Biomed Res Int 2019;2019:7196535.

8. Demaria $M$, Giorgi $C$, Lebiedzinska $M$, et al. A STAT3mediated metabolic switch is involved in tumour transformation and STAT3 addiction. Aging (Albany NY) 2010;2:823-42.

9. Tammineni P, Anugula C, Mohammed F, et al. The import of the transcription factor STAT3 into mitochondria depends on GRIM-19, a component of the electron transport chain. J Biol Chem 2013;288:4723-32.

10. Dietze EC, Chavez TA, Seewaldt VL. Obesity and TripleNegative Breast Cancer: Disparities, Controversies, and Biology. Am J Pathol 2018;188:280-90.

11. Zhang T, Zhu X, Wu H, et al. Targeting the ROS/PI3K/ AKT/HIF-1 $\alpha / \mathrm{HK} 2$ axis of breast cancer cells: Combined administration of Polydatin and 2-Deoxy-d-glucose. J Cell Mol Med 2019;23:3711-23.

12. Abdelwahed KS, Siddique AB, Mohyeldin MM, et al. Pseurotin A as a novel suppressor of hormone dependent breast cancer progression and recurrence by inhibiting PCSK9 secretion and interaction with LDL receptor. Pharmacol Res 2020;158:104847.

13. Guo C, Zhang Y, Nie Q, et al. SQSTM1/ p62 oligomerization contributes to $\mathrm{A} \beta$-induced inhibition of Nrf2 signaling. Neurobiol Aging 2021;98:10-20.

14. Vagia E, Mahalingam D, Cristofanilli M. The Landscape of Targeted Therapies in TNBC. Cancers (Basel) 2020;12:916.

15. Hoy AJ, Balaban S, Saunders DN. Adipocyte-Tumor Cell Metabolic Crosstalk in Breast Cancer. Trends Mol Med 2017;23:381-92. 
16. D'Esposito V, Liguoro D, Ambrosio MR, et al. Adipose microenvironment promotes triple negative breast cancer cell invasiveness and dissemination by producing CCL5. Oncotarget 2016;7:24495-509.

17. Wang T, Fahrmann JF, Lee H, et al. JAK/STAT3Regulated Fatty Acid $\beta$-Oxidation Is Critical for Breast Cancer Stem Cell Self-Renewal and Chemoresistance. Cell Metab 2018;27:136-50.e5.

18. Sun H, Zou J, Chen L, et al. Triple-negative breast cancer and its association with obesity. Mol Clin Oncol 2017;7:935-42.

19. Chen Y, Niu J, Li L, et al. Polydatin executes anticancer effects against glioblastoma multiforme by inhibiting the EGFR-AKT/ERK1/2/STAT3-SOX2/Snail signaling pathway. Life Sci 2020;258:118158.

20. Zou J, Yang Y, Yang Y, et al. Polydatin suppresses proliferation and metastasis of non-small cell lung cancer cells by inhibiting NLRP3 inflammasome activation via NF- $\kappa$ B pathway. Biomed Pharmacother 2018;108:130-6.

21. Samra YA, Abdelghany AM, Zaghloul RA. Polydatin gold nanoparticles potentiate antitumor effect of doxorubicin in Ehrlich ascites carcinoma-bearing mice. J Biochem Mol Toxicol 2021;35:e22869.

22. Zhao W, Chen Z, Guan M. Polydatin enhances the chemosensitivity of osteosarcoma cells to paclitaxel. J Cell Biochem 2019;120:17481-90.

23. Song X, Zhou X, Qin Y, et al. Emodin inhibits epithelial-mesenchymal transition and metastasis of triple negative breast cancer via antagonism of CC-chemokine ligand 5 secreted from adipocytes. Int J Mol Med 2018;42:579-88.

24. Zunica ERM, Yang S, Coulter A, et al. Moringa Oleifera Seed Extract Concomitantly Supplemented with Chemotherapy Worsens Tumor Progression in Mice with Triple Negative Breast Cancer and Obesity. Nutrients 2021;13:2923.

Cite this article as: Liu M, Li Y, Kong B, Zhang G, Zhang Q. Polydatin down-regulates the phosphorylation level of STAT3 and induces pyroptosis in triple-negative breast cancer mice with a high-fat diet. Ann Transl Med 2022;10(4):173. doi: 10.21037/ atm-22-73
25. Wu J, Zhu Y, Luo M, et al. Comprehensive Analysis of Pyroptosis-Related Genes and Tumor Microenvironment Infiltration Characterization in Breast Cancer. Front Immunol 2021;12:748221.

26. Xu D, Ji Z, Qiang L. Molecular Characteristics, Clinical Implication, and Cancer Immunity Interactions of Pyroptosis-Related Genes in Breast Cancer. Front Med (Lausanne) 2021;8:702638.

27. Chen D, Ma Y, Li P, et al. Piperlongumine Induces Apoptosis and Synergizes with Doxorubicin by Inhibiting the JAK2-STAT3 Pathway in Triple-Negative Breast Cancer. Molecules 2019;24:2338.

28. Kang DY, Sp N, Kim DH, et al. Salidroside inhibits migration, invasion and angiogenesis of MDA-MB 231 TNBC cells by regulating EGFR/Jak2/STAT3 signaling via MMP2. Int J Oncol 2018;53:877-85.

29. Yan H, Luo B, Wu X, et al. Cisplatin Induces Pyroptosis via Activation of MEG3/NLRP3/caspase-1/GSDMD Pathway in Triple-Negative Breast Cancer. Int J Biol Sci 2021;17:2606-21.

30. Dodington DW, Desai HR, Woo M. JAK/STAT Emerging Players in Metabolism. Trends Endocrinol Metab 2018;29:55-65.

31. Liu Q, Liang X, Liang M, et al. Ellagic Acid Ameliorates Renal Ischemic-Reperfusion Injury Through NOX4/JAK/ STAT Signaling Pathway. Inflammation 2020;43:298-309.

32. Cao F, Tian X, Li Z, et al. Suppression of NLRP3 Inflammasome by Erythropoietin via the EPOR/JAK2/ STAT3 Pathway Contributes to Attenuation of Acute Lung Injury in Mice. Front Pharmacol 2020;11:306.

33. Regua AT, Aguayo NR, Jalboush SA, et al. TrkA Interacts with and Phosphorylates STAT3 to Enhance Gene Transcription and Promote Breast Cancer Stem Cells in Triple-Negative and HER2-Enriched Breast Cancers. Cancers (Basel) 2021;13:2340. 